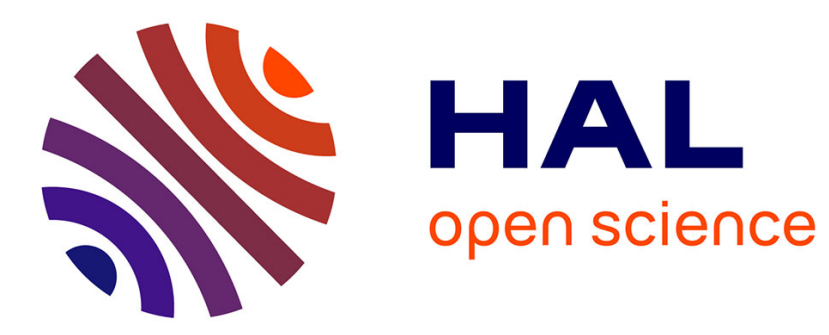

\title{
Intracavity laser spectroscopy of highly excited molecular states
}

L. Sinitsa

\section{To cite this version:}

L. Sinitsa. Intracavity laser spectroscopy of highly excited molecular states. Journal de Physique IV Proceedings, 1994, 04 (C4), pp.C4-629-C4-634. 10.1051/jp4:19944168 . jpa-00252625

\section{HAL Id: jpa-00252625 https://hal.science/jpa-00252625}

Submitted on 1 Jan 1994

HAL is a multi-disciplinary open access archive for the deposit and dissemination of scientific research documents, whether they are published or not. The documents may come from teaching and research institutions in France or abroad, or from public or private research centers.
L'archive ouverte pluridisciplinaire HAL, est destinée au dépôt et à la diffusion de documents scientifiques de niveau recherche, publiés ou non, émanant des établissements d'enseignement et de recherche français ou étrangers, des laboratoires publics ou privés. 


\title{
Intracavity laser spectroscopy of highly excited molecular states
}

\author{
L.N. SINITSA
}

Institute of Atmospheric Optics SB RAS 1, Academicheskii Av., Tomsk 634055, Russia

\section{INTRODUCTION}

The spectroscopic data for highly excited vibrational-rotational states of molecules form the information base for solving the inverse problems on the determination of the intra- and intermolecular potentials, parameters of dipole moment. The paper presents the investigation of highly excited rotation-vibration states which has been fulfild using intracavity laser spectrometers at the Institute of Atmospheric Optics.

\section{INTRACAVITY LASER SPECTROMETERS}

The method of broad band intracavity laser absorption (ICLA) spectroscopy suggested in 1970 [1] consists of quenching the laser emission at the absorption line frequencies of the species placed in a broad-band laser cavity, the generation band width of such a laser beeing much greater than the halfwidth of spectral line studied. In this case the laser emission spectrum has sharp gaps at the frequencies of absorption lines, which can be recorded with an ordinary spectroscopic instrumentation. In the intracavity laser spectroscopy a laser itself is a nonlinear detector of weak absorption.

In case when one can neglect the spectral behavior of the gain coeffitient and contribution of spontaneous radiation at the stage of quasi-continuous generation the ICLA spectrum in the vicinity of an spectral line normalized by its maximum can be described as follows.

$$
\begin{gathered}
J(v, t)=U(v, t) / U_{m}(t)=J\left(v, t_{0}\right) \exp \left(-k(v) L_{e f f}\right) \\
L_{e f f}=c t L_{a} / L_{c}
\end{gathered}
$$

where $J\left(v, t_{0}\right)$ represents the normolized spectrum formed at the initial time of generation $t_{0}, U(v, t)$ is the radiation power density at the frequency $v$ and at time $t ; U_{m}(t)$ is the value of $U(v, t)$ at frequencies off the absorption line; $L_{a}$ is the length of the absorbing layer and $L_{c}$ is the length of the laser cavity. If one neglects the pregeneration stage then it is possible to assume that $U\left(v, t_{0}\right)=1$ and hence the depth of a gap in the ICLA spectrum is determined by Bouguer law. That means that the laser, in this sense, imitates a multipass absortion cell, but with much greater effective length $L_{e f f}$ of the absorbing layer than in a simple multipass cell where it is limited by radiation losses on mirrors. In this case $L_{e f f}$ is proportional to the duration $t$ of continuous generation in the region of an absorption line studied, $L_{e f}=c t q$, where $c$ is a speed of light, $q$ is a filling factor for resonator filled with the substance under study. Pulse duration of the 7 aser $0.1 \mathrm{~ms}$ provides a sensitivity to absorption $10^{-7} \mathrm{~cm}^{-1}$ that corresponds to use $30000 \mathrm{~m}$ path length in classical spectroscopy.

The set of broad band lasers with long pulses of generation had been developed and high sensitive ICLA-spectrometers had been constructed at the IAO. The parameters of the spectrometers are presented in Table 1.

The spectrometers consist of two part : a) 1 aser with cell inside the cavity and b) grating spectometer for recording of laser emission spectra. Nd-glass and dye lasers are pumped by flash lamps. Colour center lasers are operated at 
room temperature; the $\mathrm{F}_{2}^{+}$:LiF 1 aser is pumped by Ruby laser and the $\mathrm{F}_{2}^{-}$: LiF laser - by Nd-glass laser.

Table 1. Parameters of ICLA-Spectrometers $\left(\mathrm{cm}^{-1}\right)$

\begin{tabular}{cccccc} 
Laser & $\begin{array}{c}\text { Spectral } \\
\text { Range }\end{array}$ & $\begin{array}{c}\text { Sensitivity } \\
\text { to } \\
\text { absorption }\end{array}$ & $\begin{array}{c}\text { Spectral } \\
\text { resolution }\end{array}$ & $\begin{array}{c}\text { Spectral range } \\
\text { per pulse }\end{array}$ \\
\cline { 5 - 5 } Nd-glass & $9100-9520$ & $10^{-8}$ & 0.05 & 50 \\
Nd-glass & $7350-7400$ & $10^{-7}$ & 0.08 & 30 \\
Dye & $16500-16900$ & $10^{-7}$ & 0.05 & 30 \\
$F_{2}^{-}$:LiF & $8000-9100$ & $10^{-7}$ & 0.08 & 200 \\
$F_{2}^{+}$:LiF & $10400-11000$ & $10^{-7}$ & 0.08 & 200
\end{tabular}

\section{EXPERIMENTAL STUDIES OF HIGHLY EXCITED MOLECULAR STATES}

High sensitive ICLA spectrometers allowed the recording of several thousands new atmospheric absorption lines to be performed in the spectral range above $7000 \mathrm{~cm}^{-1}$. Some tens of earlier unknown vibration - rotational bands of $\mathrm{H}_{2} \mathrm{O}, \mathrm{CO}_{2}, \mathrm{~N}_{2} \mathrm{O}, \mathrm{CH}_{4}, \mathrm{C}_{2} \mathrm{H}_{2}, \mathrm{NH}_{3}, \mathrm{HBr}, \mathrm{H}_{2}$ molecules and their isitopomers have been also recorded with these devices.

For example one can see in Fig.l the vibration-rotation bands of atmospheric and contaminating gases, the fine structure of which was studied using high-resolution ICLA spectrometers in the range $8000 \ldots 11000 \mathrm{~cm}^{-1}$.

2.1 Linear molecules

In absorption spectra of linear molecules there were recorded new vibration-rotation bands with $\Delta v \geqslant 4$ and rotational structure of Q-branches was resolved. The Fermi-resonance 2003 triad was investigated for different isotopomers of the carbon dioxide. As a results of solving the inverse spectroscopic problem the vibrational transition were identified and spectroscopic parameters of upper vibrational states were determined.

\subsection{Electric field induced transitions}

Electric field induced transitions of $\mathrm{H}_{2}$ have been investigated in strong Electric field $(100000 \mathrm{~V} / \mathrm{cm})$ using ICLA spectrometers. Energy levels as well as matrix elements of polarisability of upper vibrational state were determined for $V=2$ of the ground electronic state.

2.3 Molecular spectra at high temperature

The use of high sensitive ICLA spectrometers in combination with the effective methods for exciting and populating high vibration-rotation states of molecules enables one to obtain the information on the structure of upper 
vibration-rotation states and about the processes of energy transfer in the molecules.

Water vapor isotopomers have been investigated at temperatures $600-800 \mathrm{~K}$. An additional information on weak rotation-vibration bands including "hot" bands was obtained.

\subsection{Nonresonance laser exitation}

The investigation of molecules nonresonantly excited by a laser radiation was carried out with an intracavity laser spectrometer. In these experiments the radiation of a $\mathrm{CO}_{2}$ laser emitting $1 \mathrm{~ms}$ duration pulses of $5 \mathrm{~J}$ energy was focused on a target $\mathrm{placed} 5$ to $10 \mathrm{~mm}$ off the axis of a neodymium glass 1 aser resonator. For targets there were used samples made of metals, graphite and organic substances. Focused $\mathrm{CO}_{2}$ laser radiation produces the plasma. The expanding plasmoid thermally excites the surrounding air on the axis of the ICLA spectrometer. If neodymium glass laser is synchronized with the $\mathrm{CO}_{2}$ laser generation then one can study the absorption of these heated gases inside the ICLA spectrometer. Because of the spectral memory effect in the ICLA spectrometer the gap in the spectrum appeared at the beginning of a 7 aser pulse keeps unchanged to the pulse end, allowing thus the recording of weak induced absorption to be performed.

When laser radiation is focused onto an organic target there appear a lot of spectral lines in the spectrum (more than 200 within an interval of $100 \mathrm{~cm}^{-1}$ width), which mostly belong to the vapors of the target substance. Large values of the absorption coefficients of the observed lines $\left(" \sim 10^{-3} \mathrm{~cm}^{-1}\right)$ clearly demonstrate the possibility of making the target surface analysis using the ICLA laser spectroscopy method providing high concentration sensitivity.

In the case of metallic targets there appear not so many lines in the spectrum and they are less intensive. As the analysis has shown, the spectrum mainly contains the water vapor absorption lines due to the transitions between the upper vibration-rotation states.

\subsection{Resonance optical excitation}

A more efficient populating of the upper vibrational states of molecules can be achieved by resonance optical excitation, when the laser radiation frequency coincides with that of transition $v^{\prime \prime} j " \rightarrow v^{\prime} j^{\prime}$. In this case the laser radiation excites the molecules from the rotational level $j^{n}$ of the lower vibrational state $v^{\prime \prime}$ to the rotational level $j^{\prime}$ of the upper vibrational state $v^{\prime}$ (Fig.2). Molecular collisions result in relaxation of the molecular ensemble to the Boltzmann equilibrium distribution over rotational levels at a rate $1 / \tau_{R}$. The vibrational relaxation of molecules takes place at a rate $1 / \tau_{V}$, where $\tau_{R}$ and $\tau_{V}$ are the time constants of the rotational and vibrational relaxations, respectively.

Formation of a gap in the ICLA spectrum at the resonance optical excitation is determined by the duration $\tau_{p}$ of the laser resonance pumping, rotational relaxation time $\tau_{R}$, vibrational relaxation time $\tau_{V}$ and by the duration of spectrometer laser pulse $\tau_{L}$. The rotational relaxation time is of the order of $10^{-11} \mathrm{~s}$, while that of the vibrational one is $\sim 10^{-6} \mathrm{~s}$. The use of the ICLA spectrometer with optical excitation can be efficient if high sensitivity of measurements is assured, that is the duration of the spectrometer laser pulse must be $10^{-4}$ to $10^{-3} \mathrm{~s}$; this exceeds the rotational and vibrational relaxation times of molecules.

In the case of long excitation pulses $\left(\tau_{p}>\tau_{v}\right)$ the formation of gaps in the spectrum can be divided into the two stages. The first one occurs at a constant value of the absorption coefficient during the excitation pulse and the second one at the exponentially decreasing absorption after the excitation pulse termination. Thus the ICLA spectrum in this case can be described as follows:

$$
J(v, t)=J(v, 0) \exp \left(-\mathrm{cK}_{0} \tau_{p}\right) \exp \left(-\mathrm{cK}_{0} \tau_{\mathrm{p}}\left(1-\exp \left(-\mathrm{t}^{\prime} / \tau_{v}\right)\right)\right)
$$

where $t^{\prime}$ is counted from the moment of the laser excitation termination. 
The following peculiarities are characteristic of the ICLA spectroscopy method with the resonance optical excitation: - high sensitivity of the ICLA spectroscopy does not require high population of the intermediate levels and as a consequence there is no need for high power of exciting radiation to detect the induced absorption, - wide spectral range of ICLA spectrometers allows one to record simultaneously the whole bands due to transitions both from the excited vibrational states populated by the resonance optical radiation and from lower vibrational states populated due to vibrational relaxation.

\subsection{The HEL-resonance in the $\mathrm{H}_{2} \mathrm{O}$ molecule}

Analysis of the spectra of $\mathrm{H}_{2}^{16} \mathrm{O}$ and $\mathrm{H}_{2}^{18} \mathrm{O}$ in $1 \mathrm{mkm}$ region and solution of the inverse spectroscopic problem were carried out in specific conditions occurring in the high frequencies range, viz., large number of resonance (about 30) between the interacting levels, and also where there was a lack of spectroscopic information making the use of traditional spectrum identification methods impossible.

An analysis of the energy structure of the upper polyads indicates the existing of resonance interactions of a new type, and the new accidental resonance has a systematic character. Beginning with a certain vibrational energy it couples all the resonance polyads and should be taken into account in analyzing the absorption spectra and energy structure of highly excited vibration-rotation states of the water molecule (Fig.3). The found local resonance for the highly excited vibrational states is accociated with a strong centrifugal effect, which manifests itself in the excitation of bending vibration of large amplitude. It is due to the great magnitude of the matrix elements of the inverse moment of inertia. In this sense, the new resonance differs qualitatively from the accidental Coriolis-Darling-Dennison, and Fermi resonances considered earlier and associated with the anharmonic and Coriolis constants. A new resonance couples the states with large variations of the quantum numbers $\Delta V_{2} \approx 5$.

In accounting for the new accidental resonance, the scheme of constructing the resonance polyads for the water molecule is not valid. The highly excited vibrational states are effectively mixed together as a consequence of the strong centrifugal effect, forming the overall reservoir of vibrational states which determines the physical and chemical properties of the molecule.

Accounting for the peculiarities in the manifestation of this resonance, namely its local character and its existence only for highly excited states, it can be called an HEL (highly excited local) resonance. Similar resonance interactions associated with a strong centrifugal effect are likely to be between the highly excited states in isotope-substituted molecules of $\mathrm{H}_{2}^{17} \mathrm{O}$, $\mathrm{H}_{2}^{18} 0$, HDO, or in water-like molecules $\mathrm{H}_{2} \mathrm{~S}, \mathrm{H}_{2} \mathrm{Se}$, etc., which possess vibration of large amplitude.

\section{REFERENCE}

[1] L.A.Pakhomycheva,E.A.Sviridenkov, et, .al.Zh.Eksp.Teor.Fiz. Pis'ma Red. No.12, p.60, 1970. [Sov.JETP v;12, p.43, 1970].

\footnotetext{
The work is done with financial support of Russian Foundation for fundamental research (contract 93-02-3410)
} 
C4-633

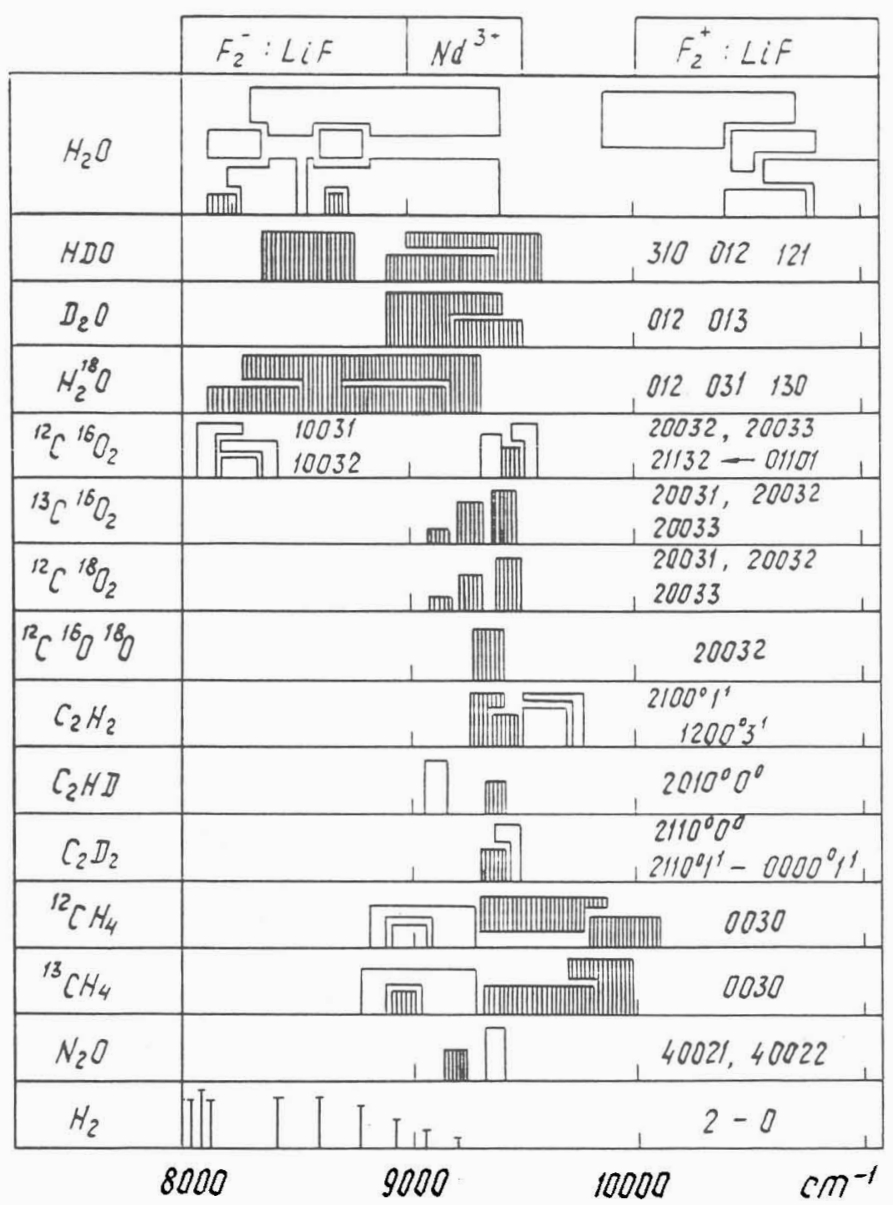

Fig.1. Rotation-vibration bands in the region $8000 \ldots 11000 \mathrm{~cm}^{-1}$ studied with the ICLA spectometers (dashed portions show newly recorded absorption bands). 


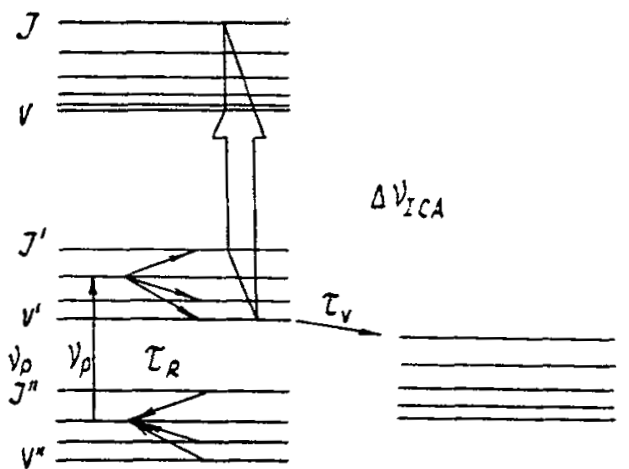

Fig.2. Molecular transitions contributing to the ICLA spectra at resonance optical excitation.

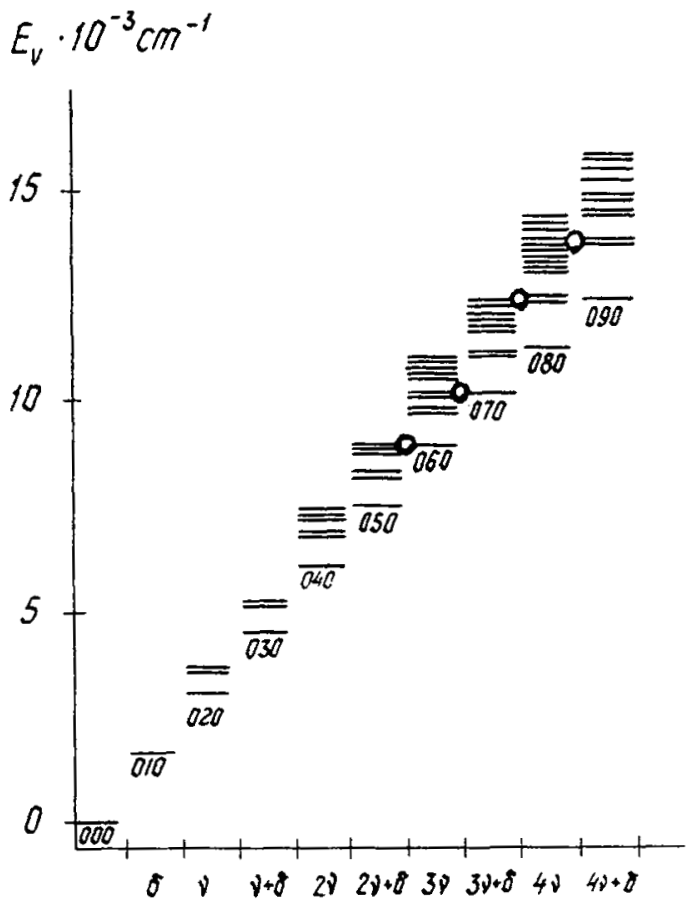

Fig.3. Diagrams of vibrational polyads of $\mathrm{H}_{2} \mathrm{O}$ molecule. o - HEL-resonance. 